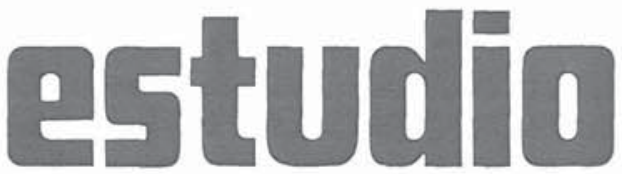

\section{sobre la adherencia de las armaduras galvanizadas en la construcción de hormigón armado}

A. J VAZQUEZ VAAMONDE, Dr. en Quimica Industrial Investigador Científico del CENIM

$447-1$

\section{sinopsis}

En este articulo se discute la necesidad de una pro. tección contra la corrosión de las armaduras en el hormigón, la posibilidad de realizar ésta mediante galvanización por inmersión en caliente y la trascenden. cia técnica y económica que supondría este tipo de protección. Se hace un estudio bibliográfico de los en. sayos de adherencia llevados a cabo y se presentan los realizados para nosotros en el Instituto Eduardo Torroja. Finalmente, se citan una serie de ejemplos característicos de obras en las que se emplea esta protección de las armaduras.

\section{INTRODUCCION}

La creciente exigencia de características en los materiales en general y de los materiales de construcción en particular, plantea como un problema acuciante el de la durabilidad de la obra realizada. El empleo del hormigón armado, y más recientemente el del hormigón pretensado, los han revelado como materiales de características excepcionales, ya que reúnen las excelencias de la resistencia a la compresión del hormigón y a la tracción del acero.

En la actualidad, los procedimientos artesanales de la construcción van siendo progresivamente sustituidos por métodos más ingenieriles, dentro de los cuales el empleo de elementos prefabricados constituye una técnica en auge que aúna las ventajas de un mejor control de calidad y una mayor economía en su fabricación y en la construcción propiamente dicha.
La corrosión del acero constituye, además, un problema de gran envergadura, ya que, por una parte, el medio ambiente que circunda íntimamente muchas obras es cada vez más y más corrosivo, y, por otra, los materiales se emplean cada vez más en el límite de sus posibilidades al ser sustituidos los antiguos y conservadores coeficientes de seguridad por otros más reducidos, fruto del desarrollo de la ciencia de los materiales. Esto último trae apareada la necesidad de que no tenga lugar ninguna alteración de los mismos que pueda traducirse en pérdida de resistencia.

La mejora en los materiales utilizados en la construcción con hormigón armado se ha ido realizando por diversas vías: unas, en directa relación con el hormigón, han conducido al desarrollo de nuevos tipos de hormigones y a un mejor control de la calidad, dosificación y puesta en obra de los mismos; otras, han buscado la protección de las armaduras procurando eliminar la porosidad que permite la difusión del aire y el agua a través del hormigón, bien vibrándolo, o aplicando a la estructura un recubrimiento impermeable que actúe sellando los poros de tipo termoplástico, termoestable o inorgánico (1); una tercera solución de proteger las armaduras la constituye la protección catódica de las mismas, técnica muy reciente y no exenta de ciertos problemas, y que, por tanto, no ha ganado todavía una extensión notable en su aplicación; una cuarta solución la constituye la protección de la misma armadura con un recubrimiento anticorrosivo, siendo uno de los que está encontrando mayor aceptación el de galvanización por inmersión en caliente. 


\section{CORROSION DE LAS ARMADURAS EN EL HORMIGON}

El acero se corroe en medios ácidos y neutros, originando iones ferrosos que en contacto con el oxígeno se oxidan dando óxido férrico hidratado. En un medio suficientemente alcalino, como el que existe en el hormigón, se alcanza un valor del $\mathrm{pH}=12,6$, y para estos valores de alcalinidad tiene lugar la pasivación del hierro, estado de protección de notable interés práctico, ya que permite el empleo de las armaduras desnudas en el hormigón, sin que, en condiciones normales, tenga lugar la corrosión de las mismas.

Aunque no pretendemos insistir sobre el tema, y remitimos al lector a obras más especializadas (2), (3), sí queremos señalar dos de las causas más frecuentes que pueden originar la corrosión de las armaduras: la presencia de cloruros y la carbonatación del hormigón.

Es de sobra conocido el hecho de que los iones halogenuro, al romper la película pasiva que protege al metal, dejan la superficie de éste inerme, a merced de los agentes de corrosión. Los iones halogenuro, y más concretamente los iones cloruro, pueden proceder de tres orígenes: la adición de cloruro cálcico al hormigón para acelerar el fraguado, el uso de cloruro cálcico como agente de limpieza y lucha contra las heladas en calles y carreteras, y la proximidad de las construcciones a la costa.

El primero de estos tres orígenes se halla limitado por el contenido relativamente bajo en cloruro cálcico, que permite utilizar la normalización española comparada con la de otros países; el segundo origen no tiene ninguna trascendencia al ser el nuestro un país de clima benigno, por lo que no tiene ninguna aplicación el empleo de cloruro cálcico en carreteras; la tercera fuente de iones cloruro sí tiene, por el contrario, notable importancia, como se desprende del hecho de que seamos un país peninsular e insular $y$, por lo tanto, con un gran perímetro costero; sobre este aspecto han de tenerse en cuenta además la creciente corriente de migración interior hacia la periferia y la creciente corriente turística, que inciden preferentemente en la zona costera, traduciéndose todo ello en un fuerte crecimiento del número de construcciones próximas a la orilla del mar.
La carbonatación del hormigón, o, en general, la reacción de óxidos gaseosos ácidos $-\mathrm{CO}_{2}, \mathrm{SO}_{2}$ y $\mathrm{SO}_{3}, \mathrm{NO}_{2}$, etc.- , da lugar a una disminución del pH del hormigón al intervenir en reacciones de neutralización con el hidróxido cálcico, pudiendo ocurrir así que el pH en la zona circundante a las armaduras no corresponda al dominio de pasividad y resulte, por el contrario, un ambiente propicio a la corrosión. Este tipo de reacciones tiene lugar con preferencia en garajes y aparcamientos, con atmósferas ricas en óxidos de carbono, y en zonas muy industriales, cuyas atmósferas son ricas en óxidos de nitrógeno y de azufre.

Además, estas causas pueden verse favorecidas por otras, algunas externas, como corrientes vagabundas, $y$ otras internas, que, debidas a las mismas armaduras $o$ al hormigón (fig. 1 a y 1 b), originan diferencias de potencial entre dos zonas de la armadura, dando lugar a la formación de pilas locales.

Por otra parte, debe tenerse presente que la formación del óxido de hierro tiene un efecto expansivo, ya que su volumen es tres veces superior al del hierro que lo origina, por lo que, rápidamente, tiene lugar la rotura de la protección conferida por el hormigón, quedando las armaduras al descubierto (fig. 2).

\subsection{Corrosión de las armaduras galvanizadas}

El intervalo de $\mathrm{pH}$ en el que el zinc puede actuar confiriendo protección al acero subyacente abarca desde el valor 6,5 al de 12,5 , entre los que los productos de corrosión que se forman originan una película protectora y adherente (4). De este modo, resulta que para todo el intervalo de $\mathrm{pH}$ que puede darse en el hormigón, incluyendo aquéllos en los que una carbonatación 


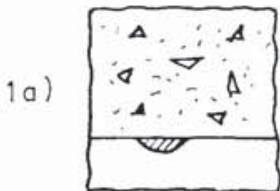

inclusiones en la superficie la superficie

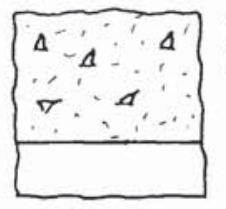

Discontinuidad en la cascarilla

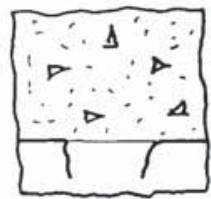

Unión de granos

1b)



Grieta de aireación diferencial

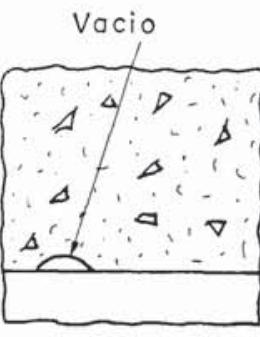

Vocios en la superficie

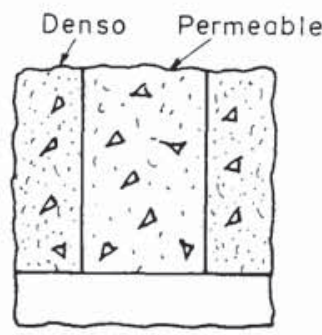

Densidad variable diferencia de humedad

Fig. 1. Diferencias de potencial debido a:

a) las armaduras:

b) el hormigón.

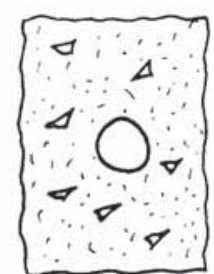

Sin corrosion

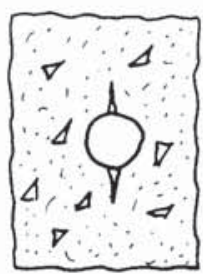

Iniciación de grietas

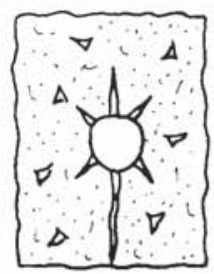

Grietas hasta la superficie

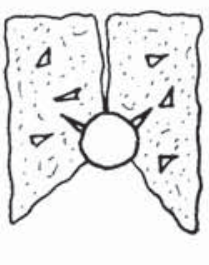

Rotura del hormigon
Fig. 2. Mecanismo de rotura del hormigón por corrosión.

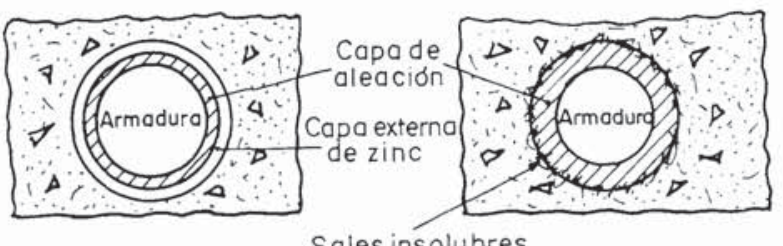

Hormigón húmedo de zinc

Hormigón fraguado

Fig. 3. Armaduras galvanizadas. profunda ha producido un descenso fuerte en el $\mathrm{pH}$ del medio, el zinc sigue manteniendo su carácter protector contra la corrosión de las armaduras (fig. 3 ).

Por otra parte, el zinc, como recubrimiento obtenido por galvanización en caliente, constituye un procedimiento adecuado para la protección contra la corrosión ambiente en medios marinos; de ahí que la presencia de cloruros, en concentraciones suficientes para provocar la despasivación del acero, no constituyan un problema grave para el zinc en cuanto a protección de las armaduras.

Finalmente, diremos que, al ser los productos de corrosión del zinc unas trece veces menos voluminosos que los del hierro, no existe la posibilidad de que tenga lugar la rotura del hormigón debido a ninguna fuerza expansiva, incluso aunque se formara una grieta que alcanzara la superficie de la armadura. 


\subsection{Ensayos de adherencia de armaduras galvanizadas}

En la bibliografía se encuentran dispersos numerosos ensayos realizados en condiciones, con materiales y métodos que no siempre les hacen fácilmente comparables entre sí. De esta situación surgió la necesidad de llevar a cabo una experimentación propia en este sentido.

Los primeros ensayos llevados a cabo quizá hayan sido los efectuados por el Bureau of Standards de los Estados Unidos en 1920. Estos ensayos, realizados por el Departamento de Barcos de Hormigón, se efectuaron sobre armaduras con 18 tipos diferentes de protección, según el procedimiento de arranque que se establece en la norma ASTM C234 (fig. 4). Se

\section{ADHERENCIA DE LAS ARMADURAS}

La base de la utilización del hormigón armado reside en la existencia de una buena adherencia entre las armaduras y el hormigón. En este sentido, hemos de señalar que la utilización de armaduras galvanizadas gozó desde siempre de un prejuicio desfavorable para su empleo en el hormigón. Este prejuicio llegó a extremos tales como el de exigir a los galvanizadores que la parte de las torres de transmisión de energía eléctrica, que se encontraban íntegramente galvanizadas, y que fueran a estar en contacto con el hormigón, se decaparan para que fuese únicamente el acero desnudo el que estuviese en contacto con el hormigón. Esto ocasionaba notables inconvenientes en cuanto a manipulación del material en la planta que, por supuesto, repercutían desfavorablemente en el coste del producto. Afortunadamente, hoy suele aceptarse, en la mayoría de los casos, que los pies de las torres estén galvanizados y embebidos en el hormigón.

Este prejuicio, que proscribía el empleo del acero galvanizado en el hormigón, se nos antoja a nosotros más fruto de la intuición que de un análisis de los resultados experimentales. Ciertamente, no puede negarse que el aspecto brillante, uniforme $y$ liso de un buen galvanizado produce una impresión de mucha peor adherencia que un acero negro, desnudo, ligeramente oxidado a la intemperie, o incluso con la cascarilla de laminación. No obstante, en éste, como en otros muchos casos, estas impresiones no se hallan de acuerdo con las realidades experimentales. necesario ejercer sobre el redondo para producir un determinado deslizamiento (5).

En la figura 5 se presentan los esfuerzos de adherencia desarrollados con cargas que producen un deslizamiento de 0,001 " en el extremo libre al cabo de 28 días de curado y también 5 meses más tarde. Aunque los resultados a 28 días son mejores para la armadura lisa, el aumento proporcional de la adherencia a 6 meses es mayor para el material galvanizado, aunque siga siendo inferior en valor absoluto (5) .

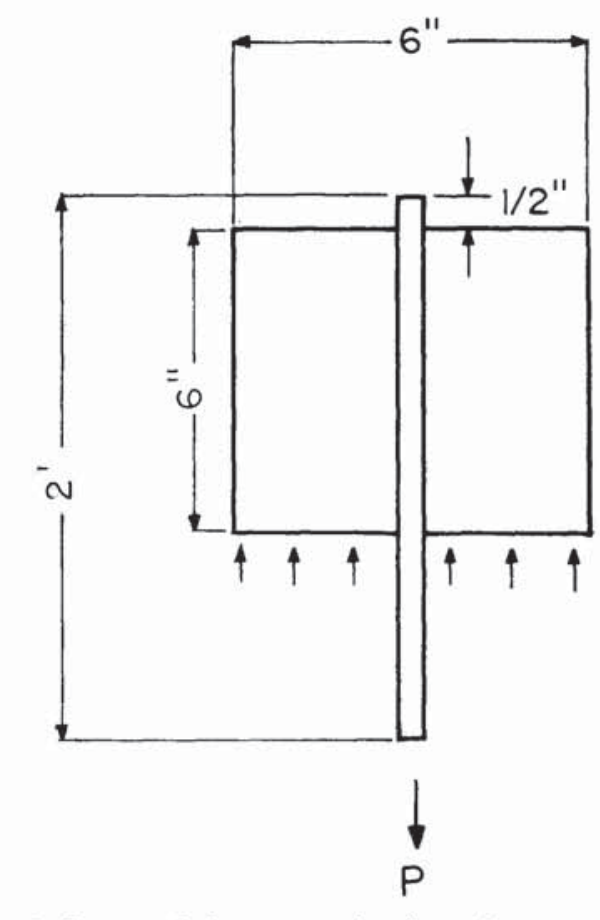

Fig. 4. Esquema de los ensayos de adherencia de las armaduras. De arrancado. 


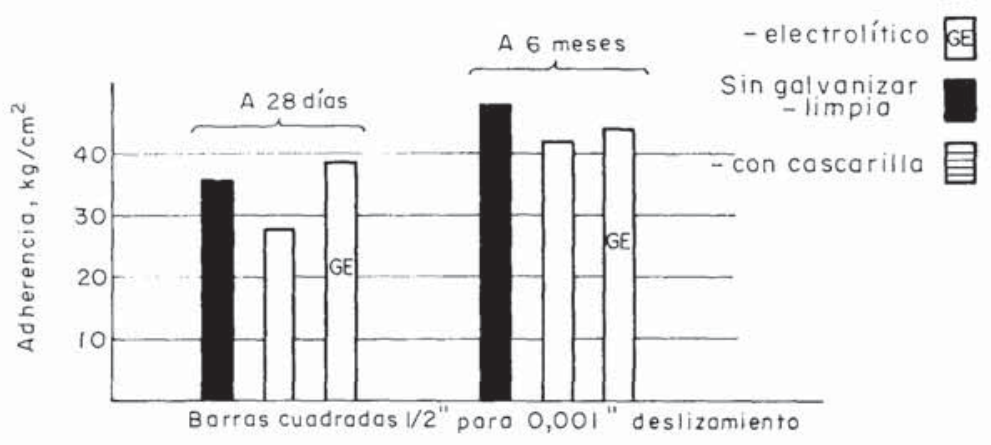

Fig. 6. British Electrical and Allied Industries Research Assn.

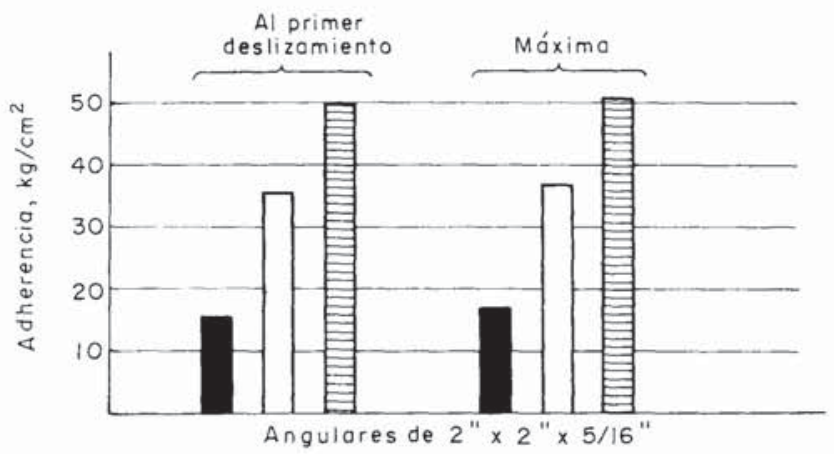

Fig. 7. Granco Steel Products Co.

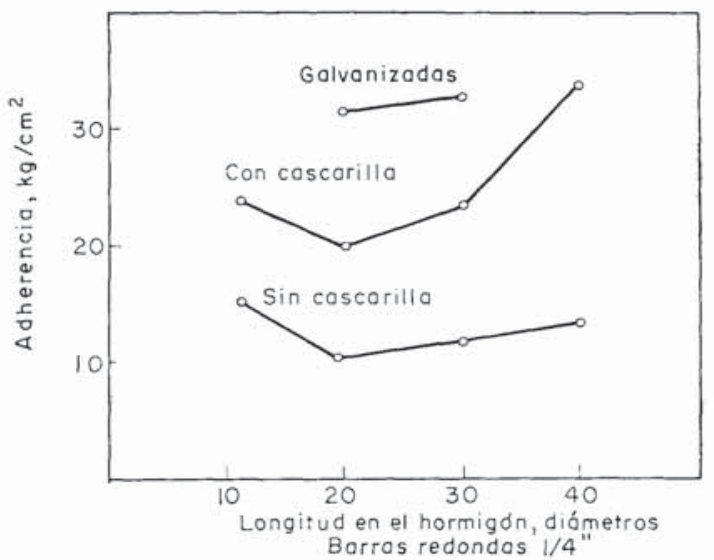

Los siguientes ensayos corresponden a una investigación efectuada por la British Electrical and Allied Industries Research Association para comprobar si se podían utilizar los pies de las torres que se iban a anclar en el hormigón, galvanizados, o, por el contrario, era necesario el uso de suplementos. En la figura 6 se presentan los resultados obtenidos en los ensayos realizados con angulares de 2 " $\times 2$ " $\times 5 / 16$ " en estado de recepción con cascarilla de laminación, limpios de cascarilla y galvanizados (5). Los resultados obtenidos para el material galvanizado son intermedios entre los correspondientes a los otros dos estados de superficie.
Posteriormente, en 1952, la Granco Steel Co. llevó a cabo una serie de ensayos utilizando armaduras de 1/4" limpias con longitudes distintas dentro del hormigón, que iban desde 12 a 40 veces el diámetro de las barras. Los resultados obtenidos con las probetas limpias, oxidadas y galvanizadas se presentan en la figura 7 (5), en las que se observan los mejores resultados para las probetas galvanizadas.

En 1954, la Oficina Federal de Ensayo de Materiales de Zurich (6) realizó, a petición de la Unión Suiza de Galvanizadores por Inmersión en Caliente, unos ensayos con armaduras de barras de $3 / 4$ " 


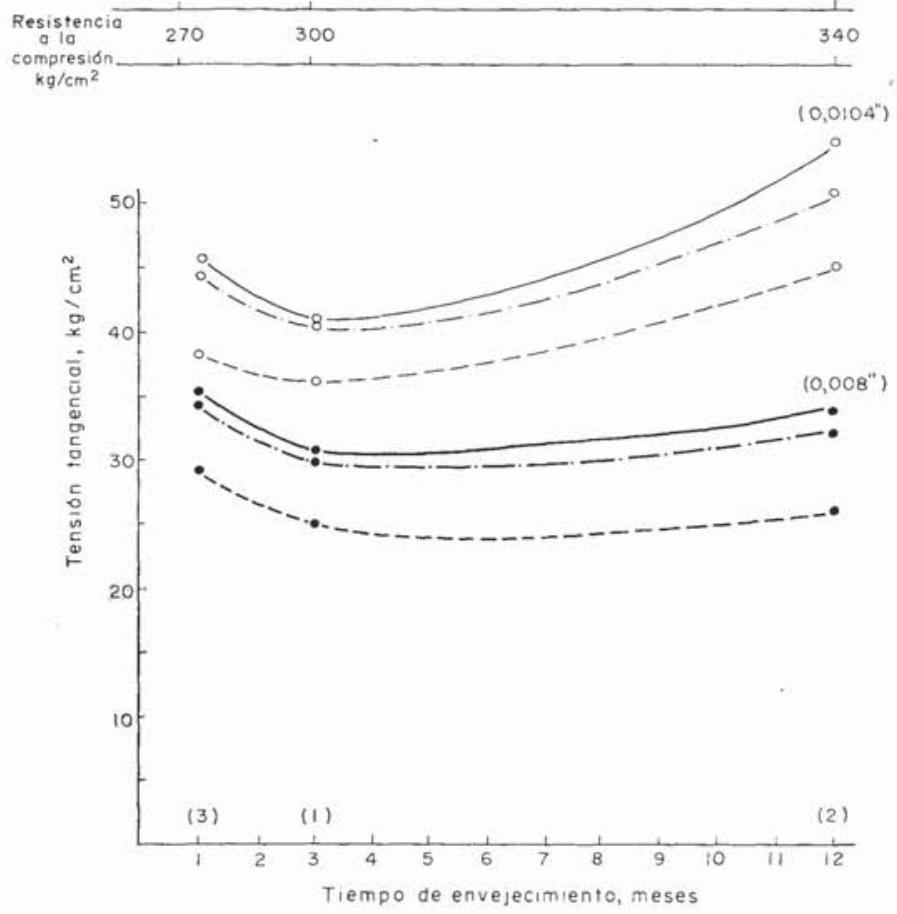

Fig. 8. Ensayos de adherencia. Tiempo de envejecimiento. Redondos, 3/4" Ø. Espesor del hormigón, 77/8".



Fig. 9. Ensayos de adherencia. Tiempo de envejecimiento. Alambres, 0,16" $\emptyset$. Espesor del hormigón, 19/16". y alambres de 0,16" de diámetro en probetas de hormigón de $77 / 8$ " y 19/16" de espesor de hormigón, respectivamente. Los resultados obtenidos en estos ensayos se presentan en las figuras 8 y 9 , siendo mejores los correspondientes al material galvanizado y apreciándose un incremento en la adherencia de todos los materiales en los ensayos de más larga duración.

En un trabajo de Frazier (7) publicado en 1970 se informa de numerosos ensayos efectuados por distintos organismos públicos, así como de los resultados obtenidos con materiales en servicio.

Robinson (8) hizo también ensayos de arrancado y de flexión en viga, pero los resultados individuales son poco numerosos - dos probetas por ensayoy presentan por ello una dispersión $\tan$ elevada que los valores medios que resultan son muy poco significativos.

Cualitativamente, se aprecia, sin embargo, que la adherencia de las probetas galvanizadas es intermedia entre las que presentan las probetas con y $\sin$ óxido.

Recientemente y a propuesta del Centro Nacional de Investigaciones Metalúrgicas, el Instituto Eduardo Torroja de la Construcción y del Cemento llevó a cabo tres series de ensayos para determinar la adherencia del hormigón sobre barras galvanizadas. Estos ensayos fueron patrocinados por la Asociación Técnica Española para el Desarrollo del Zinc (9). Los ensayos de adherencia se 


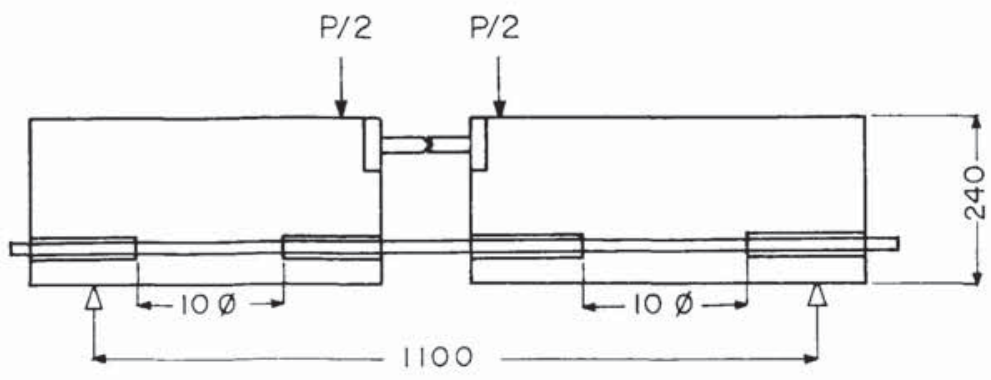

Fig. 10. Esquema de los ensayos de adherencia de las armaduras. De flexión. efectuaron después de un curado a 28 días, según el procedimiento de ensayo sobre viga $(10)$, comparándose los valores medios obtenidos sobre diez ensayos, con los que se poseían para las mismas armaduras en negro, y con los recomendados por la Comisión RILEM/FIP/CEB, "Ensayos de adherencia de aceros para armaduras de hormigón armado". Las barras que se ensayaron tenían los diámetros de 8,16 y $32 \mathrm{~mm}$ y eran corrugadas.
En la figura 11 se presentan los resultados obtenidos. Puede verse que, en algunos* ensayos, la adherencia del material galvanizado es la más elevada $(32 \mathrm{~mm} \varnothing)$, aunque en los demás la adherencia es inferior a la del acero negro, si bien en estos casos supera el mínimo exigido por las normas, lo cual demuestra la no influencia del proceso de galvanización sobre la adherencia de los aceros corrugados al hormigón. Por otra parte, se ensayaron las armaduras a tracción sin que se apreciara
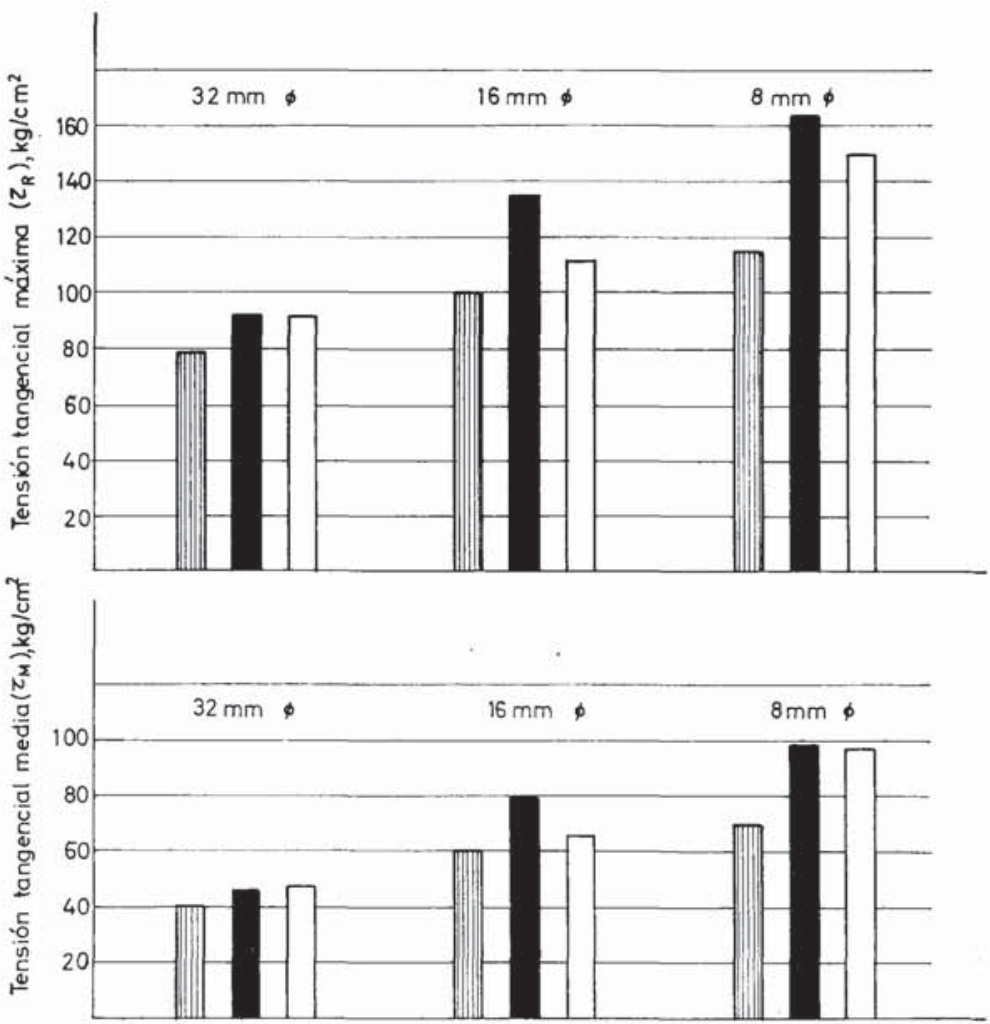

" uno de los... 
pérdida alguna de las características mecánicas del material, y a doblado sin que, para los exigidos por las normas en vigor se apreciase agrietamiento del recubrimiento.

A la vista de los resultados obtenidos parece, pues, que no hay razón para seguir manteniendo a ultranza una actitud de rechazo frente a esta nueva aplicación de los materiales galvanizados.

Recientemente, en 1970 , se ha publicado por la ILZRO la 2. ${ }^{\circ}$ edición del libro "Galvanized Reinforcement for Concrete", de gran interés para arquitectos, ingenieros de estructuras e ingenieros encargados de redactar especificaciones. En él se incluyen varios apéndices, de los que daremos nota sucinta por juzgarlos de sumo interés (10).

En el primer apéndice se informa de los resultados obtenidos en el empleo del acero galvanizado en el hormigón en las Bermudas, donde el clima marino es muy agresivo; en el segundo se citan una serie de ejemplos recientes de utilización del acero galvanizado en estructuras de hormigón armado; en el tercero se presenta una abundante bibliografía, que abarca desde 1937 a 1968, sobre la corrosión de armaduras galvanizadas; en el cuarto se comentan los resultados observados en la inspección llevada a cabo en 20 puentes construidos en hormigón armado a distancias de la costa inferiores a $500 \mathrm{~m}$; en el quinto apéndice se trata de la soldadura de las armaduras galvanizadas, recogiéndose en el sexto y último las normas ASTM más interesantes en relación con estos temas.

\section{IMPORTANCIA TECNICA Y ECONOMICA}

La trascendencia técnica y económica que puede significar para España la implantación de este procedimiento de protección de las armaduras contra la corrosión debe valorarse en un doble aspecto: el de una disminución de los costes en la construcción y/o una mejora de la calidad por un lado, y el de un aumento de la demanda del mercado interior del zinc por el otro.

\subsection{En la construcción}

Al hablar de la corrosión de las armaduras en el hormigón debe tenerse en cuenta que, en cierto modo, éste actúa como un recubrimiento protector de aquéllas; de ahí que, según el Ministerio de Obras Públicas (11), las armaduras principales no expuestas a ambientes agresivos deban estar separadas de la superficie del paramento más próximo, si éste va protegido, una distancia igual a su diámetro, siempre que éste sea superior a $1 \mathrm{~cm}$, contando esta distancia desde la parte más externa de las armaduras. En las partes curvas de las barras y si los paramentos no van protegidos y están expuestos en su superficie o en contacto permanente con agua, esta distancia mínima deberá ser de $2 \mathrm{~cm}$.

En las normas del I.E.T.c.c. (12) se establece una tabla de espesores mínimos del hormigón, según se trate de forjados y placas o de piezas lineales, para distintos grados de agresividad del ambiente (tabla 1).

\section{TABLA I}

\begin{tabular}{|c|c|c|}
\hline Agresividad exterior & $\begin{array}{l}\text { Forjados } \\
\text { y placas } \\
(\mathrm{cm})\end{array}$ & $\begin{array}{c}\text { Piezas } \\
\text { lineales } \\
(\mathrm{cm})\end{array}$ \\
\hline $\begin{array}{l}\text { Elementos } \text { abrigados de la } \\
\text { intemperie } \ldots \ldots \ldots \ldots \\
\ldots\end{array}$ & 1,5 & 1,5 \\
\hline $\begin{array}{l}\text { Idem íd., pero expuestos a } \\
\text { peligro de incendio } \ldots \ldots \ldots \ldots\end{array}$ & 1,5 & 2,5 \\
\hline $\begin{array}{l}\text { Elementos expuestos a la in- } \\
\text { temperie (heladas, lluvias } \\
\text { abundantes, etc.) ............ }\end{array}$ & 2,0 & 3,0 \\
\hline 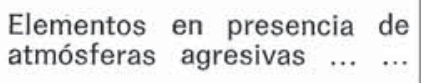 & 3,0 & 5,0 \\
\hline 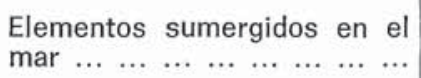 & 5,0 & 5,0 \\
\hline
\end{tabular}


Las buenas características contra la corrosión que presenta el acero galvanizado $y$ de las que hemos hablado muy sucintamente más arriba, se han traducido, de hecho, en Estados Unidos en una tolerancia de empleo de menores espesores en las construcciones que utilizan armaduras galvanizadas.

El empleo creciente de elementos prefabricados, más ligeros, se traduciría en una serie de beneficios económicos que se presentarían a lo largo de todo el ciclo de construcción. A un menor consumo de hormigón cabe añadir un menor coste del transporte por elemento, una potencia menor de las grúas necesarias para su colocación, una mayor facilidad y velocidad en la misma, lo cual significaría un menor tiempo de construcción. Finalmente, nos encontraríamos con un menor peso de la obra, lo cual se traduciría en una mayor esbeltez de los elementos empleados. Frente a estos conceptos se encuentra el de un mayor coste de las armaduras galvanizadas frente a las negras.

La disminución en el coste que puede significar una reducción en el espesor del hormigón frente al coste extra debido a la galvanización de las armaduras, tiene un sentido distinto según cuál sea el tipo de elemento que se considere. Así, en el caso de vigas, en las que las armaduras constituyen un elevado porcentaje del peso, se da, en general, un aumento neto del coste; por el contrario, en el caso de paneles prefabricados, en los que las armaduras pueden significar tan sólo un $5 \%$ del coste total, el coste extra debido a la galvanización de las armaduras es inferior a la disminución conseguida gracias al uso de espesores de hormigón menos gruesos.

En este orden de cosas resulta ilustrativo el ejemplo que presentan Cornet y Bresler sobre la utilización de armaduras galvanizadas en el tablero de un puente. El aumento en el coste debido a la galvanización de las armaduras fue equivalente al que resultaría de la restauración, de una sola vez en la vida del puente, de tan sólo el $10 \%$ de su superficie (13).

Existen además otros aspectos que deben tenerse en cuenta y que son menos susceptibles de una valoración económica en términos cuantitativos. Uno de ellos lo constituye la valoración estética de la obra. Este punto tiene un interés creciente, ya que el empleo de fachadas con hormigón visto es cada vez más frecuente $y$, naturalmente, no se puede permitir que sobre ellas aparezcan manchas de óxido. El segundo aspecto es el de la mayor garantía que significa en una obra la utilización de materiales de buenas características en cuanto a su resistencia frente a la corrosión.

\subsection{Consumo de zinc}

La ampliación del mercado del zinc que puede originar la implantación de esta técnica en la construcción es muy grande, según se pone de manifiesto en la figura 12 , en la que se presentan los consumos anuales de redondos y su incremento anual referido al año precedente. Estas cifras se han obtenido sobre la base del consumo de hormigón y tomando los coeficientes 10 para el cociente hormigón/acero

Fig. 13. Producción y consumo de zinc en España. Consumo de zinc en galvanización por inmersión en caliente.

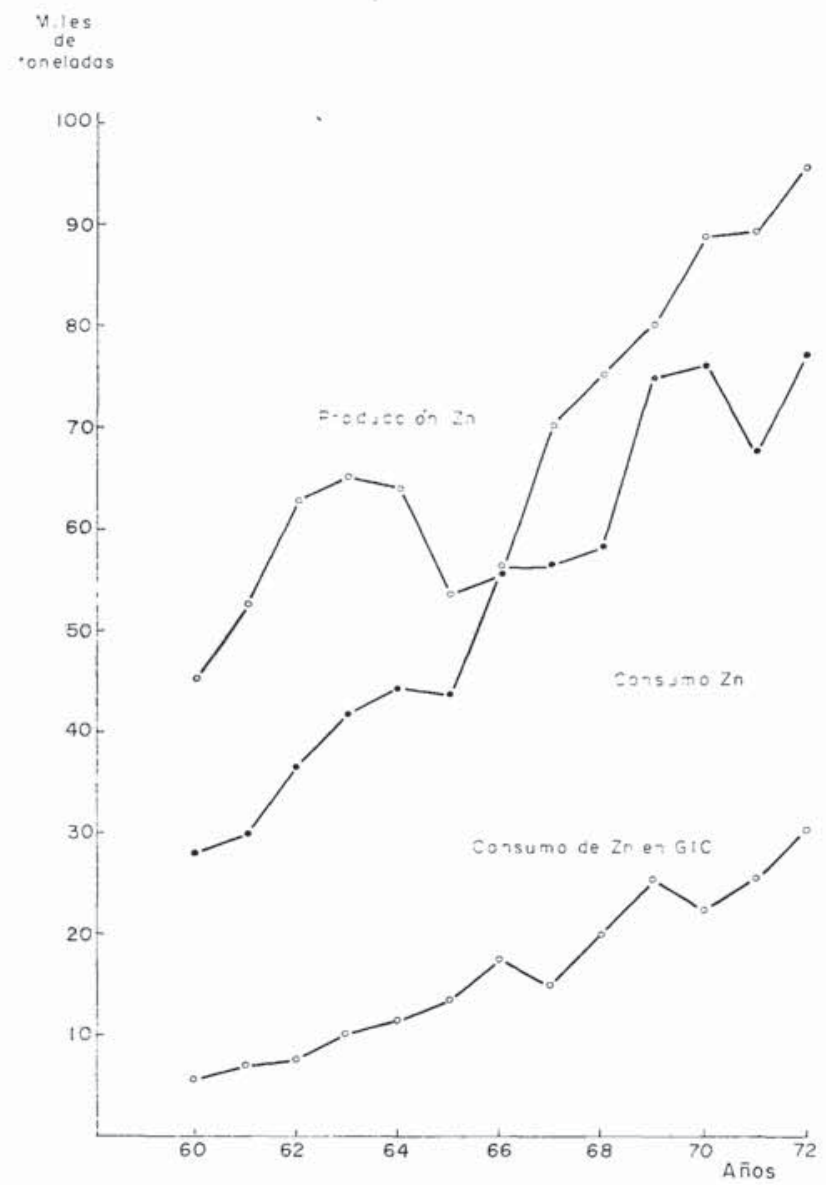


equivalente y 1,27 para el cociente acero equivalente/redondo. En estas cifras se incluyen tanto los redondos lisos como los corrugados.

Si tomamos como base de cálculo el precio de 10.000 ptas./t para los redondos, el valor del acero protegido ascendería a 12.000 millones ptas./año. El espesor habitual de un recubrimiento galvanizado es de $80 \mu \mathrm{m}$, que equivalen a $600 \mathrm{~g} / \mathrm{m}^{2}$, lo cual significa de 1 a $5 \%$ de zinc respecto al peso del redondo. Estimando en $80-85 \%$ el rendimiento de empleo de zinc en la galvanización y tomando un valor medio del 3,5\% para el peso del zinc referido al del redondo, tenemos un valor final de $4 \%$ en peso para el consumo de zinc.

Refiriéndonos al año 1971, la galvanización de todos los redondos empleados en la construcción significaría un consumo de zinc de $48.000 \mathrm{t} /$ año. Esta cifra es algo menos del doble del consumo de zinc dedicado a la galvanización por inmersión en caliente, y casi al $70 \%$ del total de zinc consumido en España (fig. 13).

Procentaje de variación anual
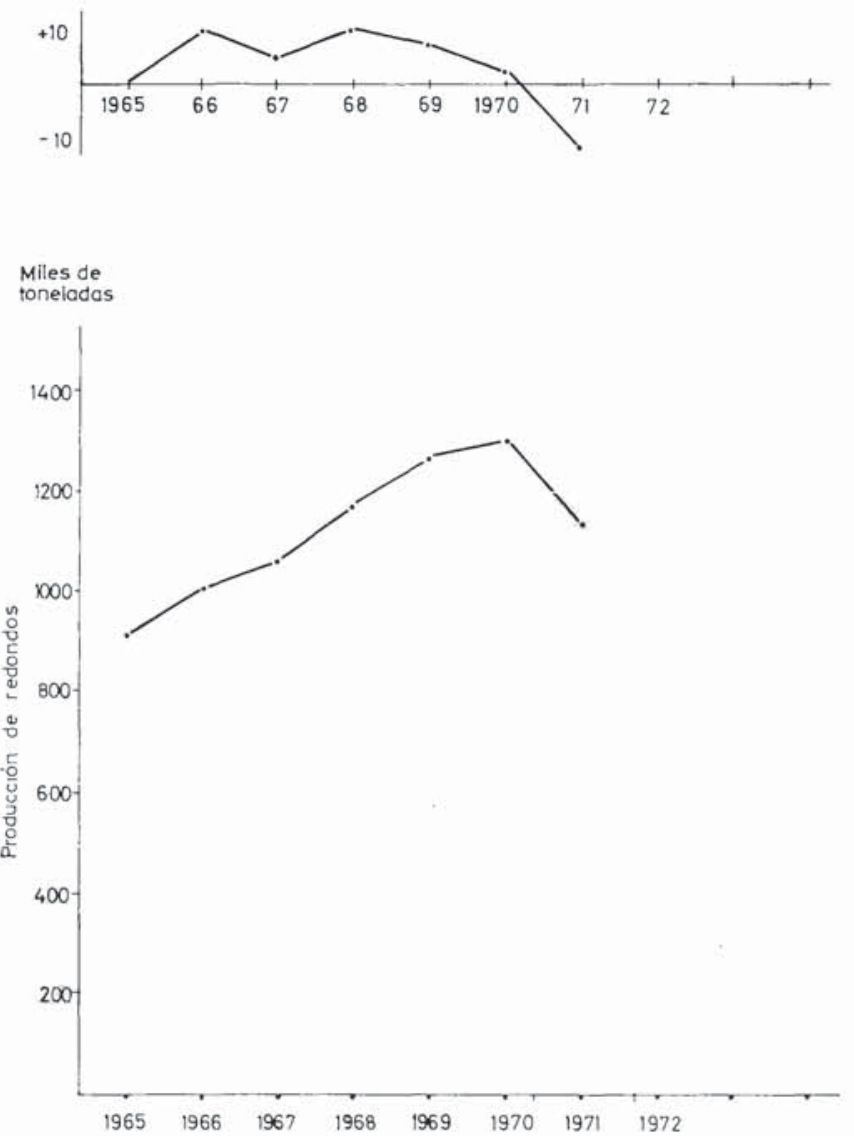

Fig. 13: Producción de redondos para armaduras.

\subsection{Ejemplos de aplicaciones}

Sin pretender tratar exahustivamente el tema, y con el único ánimo de presentar algunos ejemplos de aplicación de armaduras galvanizadas en la construcción de hormigón armado, vamos a referirnos a algunas obras, de las que señalaremos simultáneamente las características más interesantes.

Hemos dicho ya que en los Estados Unidos hace muchos años que se usan los redondos galvanizados, y hemos dicho también que en ellos se permite una reducción en el espesor del hormigón. Así, podemos señalar el caso de la construcción del Community College en la ciudad de Nueva York (14), en el que se emplearon paneles decorativos en hormigón prefabricado de color blanco brillante. El empleo de armadura y malla galvanizadas garantiza el mantenimiento de la calidad estética de los paneles, pese a que su utilización ha permitido reducir el espesor del hormigón de 11/2", que es lo preceptuado en el caso de emplear armaduras negras, a solamente 3/4" (fig. 14).

Esta misma doble razón fue la que también motivó el empleo de armaduras galvanizadas en la construcción de un banco de 15 pisos de altura en Waikiki (Hawai) (fig. 15), en donde la agresividad de la atmósfera es notable (15). Los espesores con los que se construyeron los elementos prefabricados son los mínimos compatibles con la elevada corrosión típica de una zona tropical con elevadas precipitaciones y humedad relativa. Se emplearon por ello de $21 / 2$ " a 3 ". Si las armaduras hubieran sido en negro se hubieran producido fisuras en el hormigón, por las que hubiera llegado la humedad a las armaduras, dando lugar a la migración del óxido formado hacia la superficie e, incluso, eventualmente, a la rotura del hormigón. Este problema se evitó utilizando armaduras galvanizadas de $3 / 8$ " y de $1 / 2$ " de diámetro (10). 

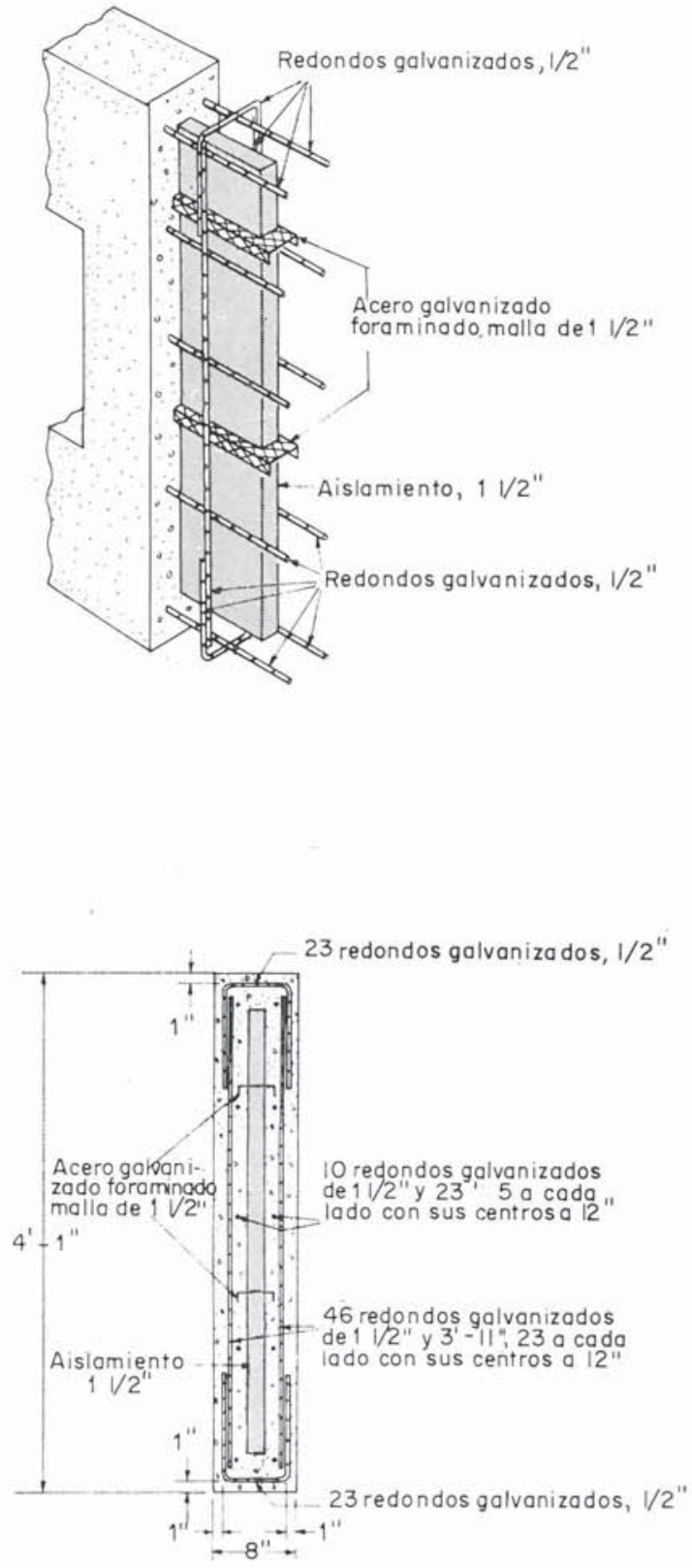

Fig. 14. Sección de un elemento prefabricado en hormigón armado con acero galvanizado.

La malla galvanizada se ha venido usando como armadura normal para los paneles que constituyen el muro exterior de edificios industriales, iglesias y un gran número de edificios públicos. En este sentido cabe destacar la cubierta laminar del Teatro de la Opera de Sidney, hermosísimo ejemplo de la estética e ingeniería de nuestros días (fig. 16). Construido a la misma orilla del mar, la cubierta está reforzada con tres mallas de acero galvanizado y lleva un recubrimiento de tejas impermeables. Entre las tejas hay solamente una delgada capa de hormigón de 1/4" de grueso protegiendo el acero galvanizado de la malla (17).

Otro ejemplo reciente de empleo lo tenemos en la construcción del "Department of Housing and Urban Development" en Washington, en el que se utilizaron armaduras galvanizadas"(siempre que se pensara en emplear espesores de hormigón) inferiores a $5 \mathrm{~cm}$ como garantía de inalterabilidad de la fachada (18). Este edificio tuvo un presupuesto de 26 millones de dólares, de los que 50.000 correspondieron al costo de las $400 \mathrm{t}$ de armaduras galvanizadas.

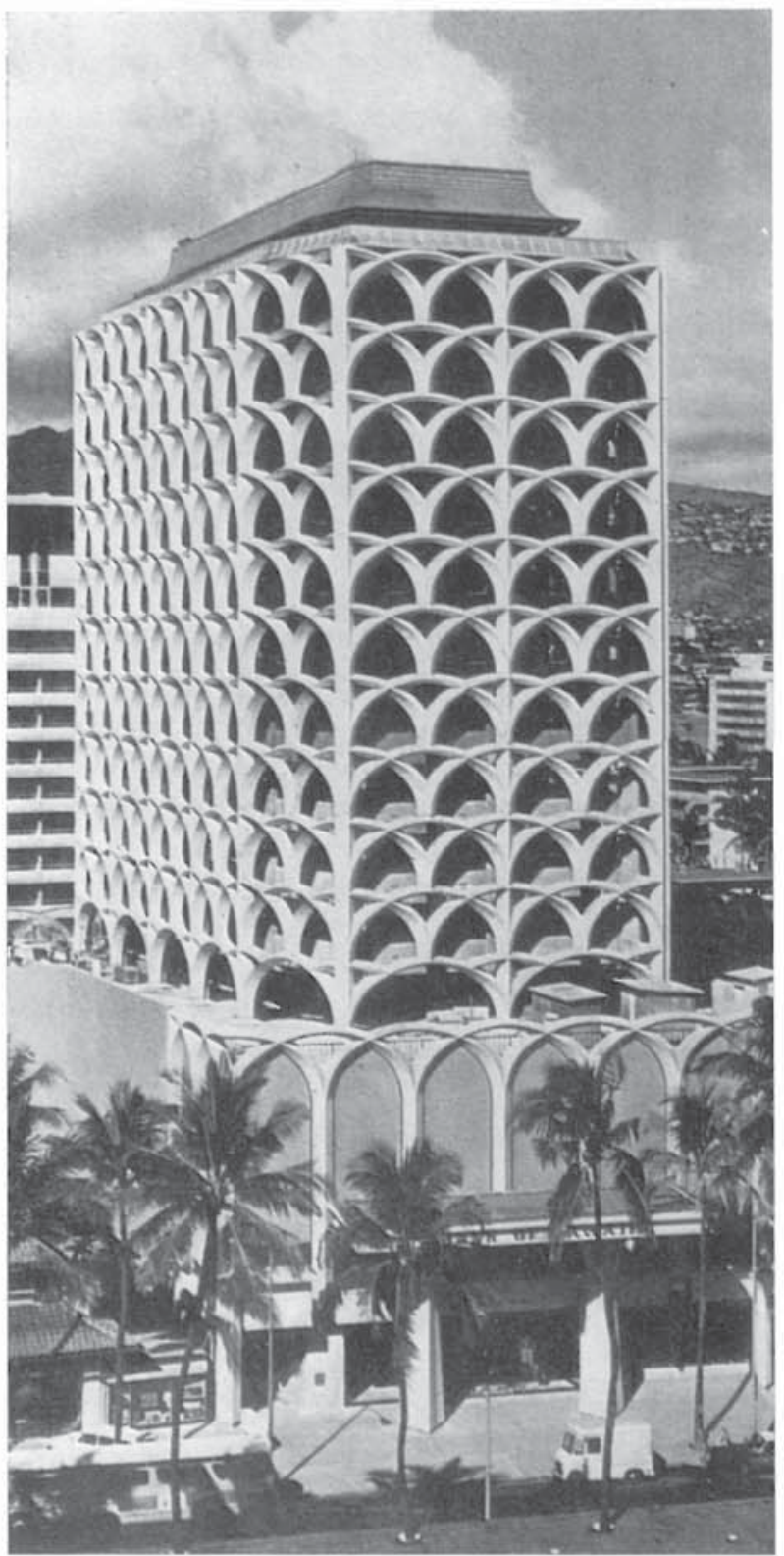

* Por error, lo impreso entre paréntesis en el texto debe leerse: dado que los espesores de hormigón eran... 


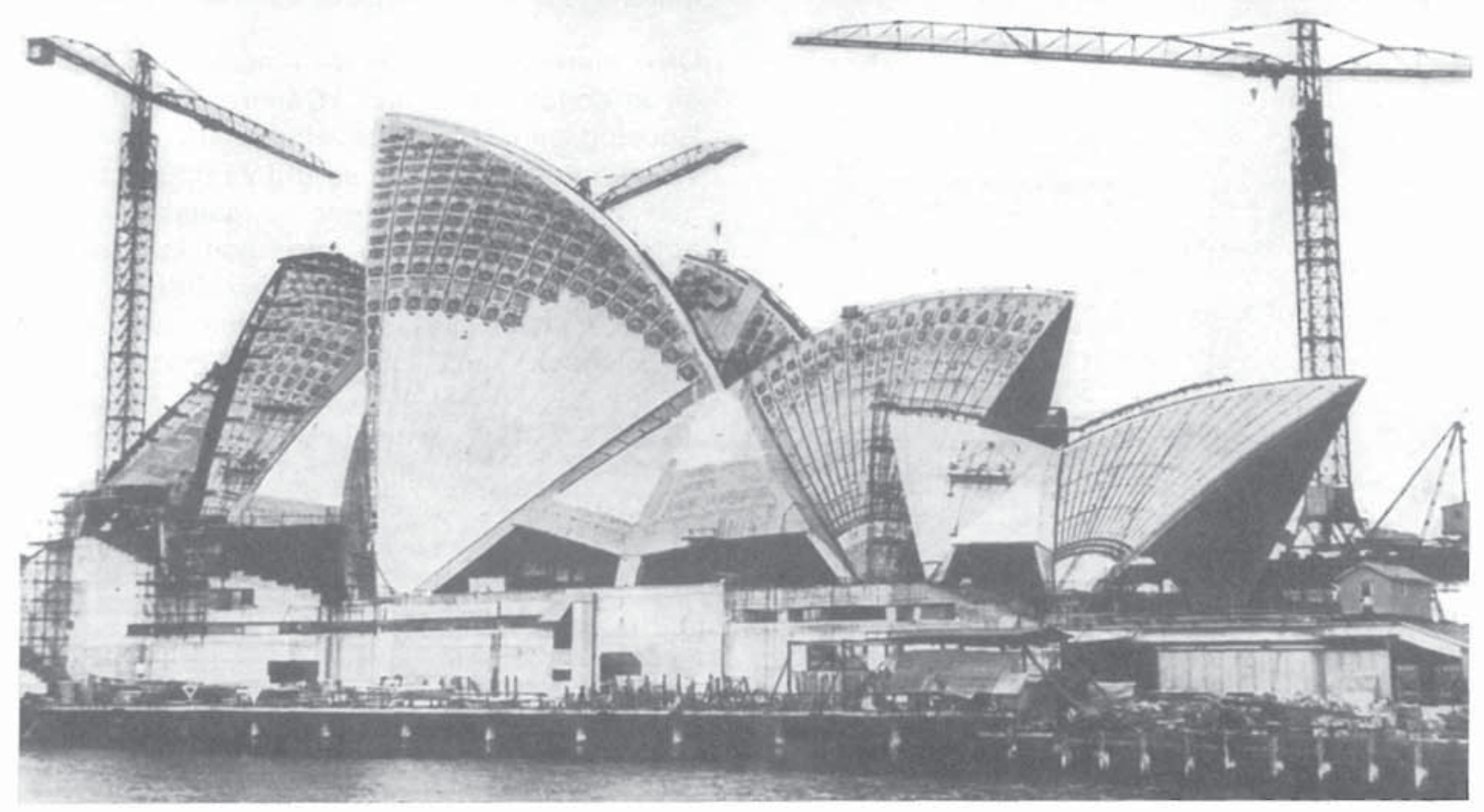

Fig. 16. Vista lateral de la Opera de Sydney en estado de construcción.

En las cubiertas de hormigón se emplearon mallas de acero galvanizado.

Recientemente, en la Universidad de Wisconsin se han construido más de 20 edificios en los que se utilizaron armaduras galvanizadas en elementos prefabricados (19). El presupuesto bianual dedicado a la construcción por el Departamento de Administración del Estado de Wisconsin es de 200 millones de dólares, de los que la mayoría emplean elementos prefabricados con armaduras galvanizadas.

Sin pretender con ello tratar exahustivamente el tema, queremos señalar también algunos ejemplos europeos. Así, tenemos los paneles de revestimiento de la Universidad de York, en los que se utilizaron barras de $5 / 16$ " y $1 / 4$ " en paneles de $13 / 4 "$ ", los cuales tenían un espesor del hormigón de tan sólo 3/4". En el Colegio Universitario de Londres se usaron armaduras de $3 / 8$ " y malla con un recubrimiento de hormigón de también sólo 3/8". En el Pabellón de Deportes de Birmingham se empleó malla galvanizada en paneles de $11 / 2$ " de gruesos (20).

El Departamento de Carreteras francés ha empleado tableros construidos con armaduras galvanizadas en la construcción del Toutry Viaduct en la autopista A6, y el Departamento de Carreteras de Quebec comenzó a utilizar el acero galvanizado en las armaduras de los viaductos en el año 1965 , siendo pionero en este tipo de aplicaciones. Otros proyectos canadienses, en los que también se usa el acero galvanizado, son el viaducto de ta carretera elevada 11 y el de autorruta 20 (21). La razón fundamental que motivó el ampleo de armaduras galvanizadas fue la de procurar que no hubiese agrietamiento ni rotura en unos elementos que iban a estar sometidos a las acciones del viento, de la lluvia y a ciclos de helada y deshielo. Por otra parte, debía tenerse en cuenta la disminución que ello significaría en los costos de mantenimiento.

\section{CONCLUSION}

Con esta publicación se pretende, además de hacer un resumen bibliográfico sobre las ventajas que pueden significar el empleo del acero galvanizado en las armaduras de las construcciones en hormigón armado, contribuir a ilustrar con datos experimentales propios sobre la buena adherencia que, al menos en estos primeros ensayos realizados a 28 días, presentan estos materiales. 


\section{BIBLIOGRAFIA}

1. J. R. CASTLEBERRY, "Corrosion Prevention for Concrete and Metal Reinforcing in the Construction Industry". Mat. Prot., marzo 1968, 21-28.

2. J. CALLEJA, "Corrosión de armaduras en los hormigones armados y pretensados». Monografía núm. 256 del I.E.T.c.c., Madrid.

3. I. CORNET y B. BRESLER, "Corrosion of Metal in Concrete: A Selected Bibliography". Rep. TS-66-6, Grant n. $44,2445-42510$, agosto 1966. Colegio de Ingeniería, Univ. California, Berkeley.

4. A. J. VAZQUEZ, "Galvanización por inmersión en caliente: Propiedades y aplicaciones". DYNA, núm. 7, 1972. 368-378.

5. 1. A. BENJAMIN, "The Adhesion of Concrete to Galvanized Steel», separata de la Am. Hot.-Dip. Gal. Assn. Inc., 10-5-61.

6. Anónimo, "Bond Strength of Galvanized Steel», separata B-13 de la AHDGA, Inc., 1961.

7. K. S. FRAZIER, "The Protection of Reinforcing Steel in Concrete", Offshore Technology Conf., separata OTC 1267, 1970.

8. K. E. ROBINSON, "The Bond Strength of Galvanized Reinforcement». Cement and Concrete Assn., Tech. Rep. TRA/220, marzo 1956.

9. Expediente 6105 del I.E.T.c.c., 1972.

10. ILZRO, Inc., "Galvanized Reinforcement for Concrete», Zinc Inst. Inc. e ILZRO, Inc., 2." Ed. N. Y., 1970.

11. M.O.P., "Instrucción para el Proyecto y Ejecución de Obras de Hormigón Armado», 1." parte, Patronato "Juan de la Ciervan del C.S.I.C., 1958.

12. I.E.T.c.c., "Instrucción para el Proyecto y Ejecución de Obras de Hormigón en Masa o Armado", art. 13.3, 1968, Ministerio de Obras Públicas.

13. I. CORNET y B. BRESLER, "Algunos desarrollos recientes en la utilización de armaduras de acero galvanizado en el hormigón", 9. conf. int. sobre Galv. en Caliente, Düsseldorf, 1970, 16 pp. Asoc. Tec. Esp. Galv.

14. J. C. B. MOORE, "Galvanized Rebar for Precast Concrete Panels", The Construction Specifier, enero 1968.

15. ANONIMO, "Interlacing Archs Give Bank a Festive Look", An Engineering News-Record, separata 9-II-67, McGraw Hill, Inc.

16. WIMBERLEY, WHISENAND, ALLISON, TONG \& GOO, Architects, Ltd., comunicación privada.

17. ANONIMO, Boletín Fe $+\mathrm{Zn}$, Boletín de la Asoc. Europ. de Galv. en Caliente, núm. 12, Ed. española, 1971.

18. P. PALOMAR COLLADO, "Moderna arquitectura del hormigón. Fachadas prefabricadas". Cemento y Hormigón, núm. 45, separata de la Agrup. Fabr. de Cemento de España.

19. R. LAUESDORF, Departamento de Ingeniería de la Universidad de Wisconsin, comunicación privada.

20. L. H. EVERETT y K. W. S. TREADAWAY, "The Use of Galvanized Reinforcement in Building", 8." conf. Int. on Hot.-Dip. Galv., Londres, 1968.

21. THE REUBEN H. DONNELLEY CO., "Maintenance Problems Cut with Galvanized Steel Reinforcement", Rural \& Urban Roads, mayo 1968.

\section{résumé}

Etude sur l'adhérence des armatures galvanisées dans la construction en béton armé

A. J. Vázquez Vaamonde, Dr. en chimie industrielle chercheur scientifique du industr

Dans cet article, l'auteur évoque le besoin d'une protection contre la corrosion des armatures dans le béton, la possibilit d'assurer cette protection par la galvanisa. tion par immersion à chaud et limportance technique et économique que supposerait e type de protection. In quit une etude coxé cutés et présente les essais effectués chón dite une serie d'exemples caracteristique pour les armatures.

\section{summary}

Study of the bonding of galvanized structures in reinforced concrete construction

Dr. A. J. Vázquez Vaamonde, Chemical Engineer CENIM Researcher

This article discusses the need for anticorrosion protection for structures in concrete, the possibility that hot-dip galvanizacis and cal and economic significance that this type bibloction would afford. There is a bibliographic study of the bonding tests carts made by us the Instituto Eduardo Torroje orroja. Lastly, a representative sampling protection was used.

\section{zusammenfassung}

Studie über die Adhäsion der Galvanisierarmierung in der Eisenbetonkonstruktion

A. J. Vázquez Vaamonde, Dr. in Industriechemie Wissenschaftlicher Forscher des

In diesem Artikel erörtert man die Notwendigkeit, die Armierung in Beton gegen Átzung zu schützen, die Möglichkeit, das Gerüst mittels Galvanisierung durch Heisseintauchung $\mathrm{zU}$ schützen und ausserdem die technische und finanzielle Bedeutung dieser Schutzart.

Es wird eine bibliographische Studie der durchgeführten Adhäsionsproben, deren $\mathrm{Er}$ gebnisse uns im aInstituto Eduardo Torro ja” vorgeführt werden, aufgestellit. Mit einer Aufzahlung von charakteristischen Beispielen von Bauten, in denen die Armie endet der Artikel. 